\title{
Identifying the implied volatility using the total variation regularization
}

Yun Liu and Xijuan Liu*

\section{"Correspondence:}

Ixj_zhen@foxmail.com

College of Information Engineering,

Tarim University, Alar, Xinjiang

843300, China

\begin{abstract}
This paper studies an inverse problem of determining the implied volatility in the financial products linked with gold price, which has important application in financial derivatives pricing. Based on the total variation regularization strategy, the existence and necessary condition of the minimum for the control function are addressed, and the local uniqueness of the solution is also given by a modified case. Furthermore, the stability and convergence for the regularized approach are discussed. The results obtained in this paper may be useful for those who engage in hedging or risk management.
\end{abstract}

Keywords: inverse volatility problem; optimal control; total variation regularization; existence; necessary condition; uniqueness; stability and convergence

\section{Introduction}

In this work, we study an inverse problem of determining the implied volatility in the financial products linked with gold price. This problem was issued by the bank of East Asia in Guangzhou (China) on February 20, 2006. The profits of this product not only depend on some cumulative indexes of gold price but also on some trigger indexes. Thus this problem can be regarded as a double barrier option question (see $[1,2])$. The mathematical model can be stated in the following form:

To simplify the problem, we make the following assumptions (see [2]):

(1) Gold price $S_{t}$ satisfies a geometric Brownian motion

$$
\frac{d S_{t}}{S_{t}}=\mu d t+\sigma(S) d W_{t}
$$

where $\mu$ is the expected rate of return, $\sigma(S)$ is the volatility, and $W_{t}$ is the standard Brownian process which satisfies

$$
E\left(d W_{t}\right)=0, \quad \operatorname{Var}\left(d W_{t}\right)=d t
$$

(2) The limit range of the gold price is $\left[S_{a}, S_{b}\right]$, and the initial price is $S_{0}$, which satisfies $S_{a} \leq S_{0} \leq S_{b}$. The upper bound $S_{a}$ and the lower bound $S_{b}$ are determined by the estimation of expectation of gold price, which are made by the bank.

(c) The Author(s) 2018. This article is distributed under the terms of the Creative Commons Attribution 4.0 International License (http://creativecommons.org/licenses/by/4.0/), which permits unrestricted use, distribution, and reproduction in any medium, provided you give appropriate credit to the original author(s) and the source, provide a link to the Creative Commons license, and indicate if changes were made. 
(3) The risk-free rate of the dollar market is a nonnegative constant $r$, and the annual rate of gold price is $r_{0}$ when the price does not hit the interval $\left[S_{a}, S_{b}\right]$. Otherwise, it is denoted by $r_{1}$.

Based on the above assumptions and the standard $\Delta$-hedging theory (see [1]), we can obtain the following Black-Scholes type equation:

$$
\frac{\partial V}{\partial t}+\frac{1}{2} \sigma^{2} S^{2} \frac{\partial^{2} V}{\partial S^{2}}+r S \frac{\partial V}{\partial S}-r V=0,
$$

where $V$ is the price of the corresponding financial product.

Under the agreement, investors can get the highest annual interest rate of $r_{0}$ if the price of gold does not touch the boundary of interval $\left[S_{a}, S_{b}\right]$ through the observation period $[0, T]$. So the termination condition is given by

$$
V(S, T)=1+r_{0} T \quad\left(S_{a}<S<S_{b}\right)
$$

If the gold price will never hit the endpoint of interval $\left[S_{a}, S_{b}\right]$ from the initial moment to time $t$, then the investor can gain the profit with the annual interest rate $r_{1}$. Considering the discount, we obtain the following boundary condition:

$$
V\left(S_{a}, t\right)=V\left(S_{b}, t\right)=\left(1+r_{1} t\right) e^{-r(T-t)} \quad(0 \leq t<T)
$$

Once the gold price passes over the superior limit or below the inferior limit of the price, the interest will no longer be calculated and the contract is stopped. It seems that the price of the financial product has the character of double barrier options. The domain for determining solution is as follows:

$$
\Omega=\left\{(S, t) \mid S_{a}<S<S_{b}, 0 \leq t<T\right\} .
$$

Based on the above analysis, the value $V(S, t)$ satisfies the following backward partial differential equation (PDE):

$$
\begin{cases}\frac{\partial V}{\partial t}+\frac{1}{2} \sigma^{2} S^{2} \frac{\partial^{2} V}{\partial S^{2}}+r S \frac{\partial V}{\partial S}-r V=0, & (S, t) \in \Omega \\ V\left(S_{a}, t\right)=V\left(S_{b}, t\right)=\left(1+r_{1} t\right) e^{-r(T-t)}, & t \in[0, T) \\ V(S, T)=1+r_{0} T, & S \in\left(S_{a}, S_{b}\right)\end{cases}
$$

For given $\sigma=\sigma(S)$, equation (1.1) is called the forward or direct problem which is known to be well posed in the sense of Hadamard (see [3]). In this paper, we are interested in the inverse problem, i.e., we shall determine the unknown volatility function $\sigma(S)$ and $V(S, t)$ simultaneously. In general, some extra condition is indispensable for the inverse coefficient problem. In this paper, we use the following extra condition:

$$
V\left(S^{*}, t^{*}\right)=V^{*}(S), \quad S \in\left[S_{a}, S_{b}\right]
$$

where $0 \leq t^{*}<T$ is the fixed observation time. It should be pointed out that the above extra condition (1.2) is just an approximate form. In fact, when the gold prices vary during the time observation domain $\left[t^{*}-\delta, t^{*}\right], \delta>0$, people may obtain various financial product 
prices $V(S, t)$ from the financial market. So, if the parameter $\delta$ is small enough (compared with $\left.T-t^{*}\right)$, the observed data $V(S, t) \in\left[t^{*}-\delta, t^{*}\right]$ can be used to approximate (1.2).

We make the following change in the variable:

$$
U=V-\left(1+r_{1} t\right) e^{-r(T-t)}, \quad x=\ln \frac{S}{S_{a}}, \quad \tau=T-t,
$$

and $a(x)=\frac{1}{2} \sigma^{2}(S)$. Then the function $U$ satisfies the following inverse parabolic problem with terminal observation:

$$
\begin{cases}U_{\tau}-a(x)\left(U_{x x}-U_{x}\right)-r U_{x}+r U=f(x, \tau), & (x, \tau) \in(0, l) \times\left(0, \tau^{*}\right], \\ U(x, 0)=\phi(x), & x \in(0, l), \\ U(0, \tau)=U(l, \tau)=0, & \tau \in\left[0, \tau^{*}\right], \\ U\left(x, \tau^{*}\right)=U^{*}(x), & x \in(0, l),\end{cases}
$$

where

$$
\begin{aligned}
& l=\ln \frac{S}{S_{a}}, \quad \tau^{*}=T-t^{*}, \quad U^{*}=V^{*}-\left(1+r_{1} t^{*}\right) e^{-r\left(T-t^{*}\right)}, \\
& \phi(x)=\left[r_{0}-r_{0}\right] T, \quad f(x, \tau)=r_{1} e^{-r \tau} .
\end{aligned}
$$

Volatility is a very important parameter in the Black-Scholes equation, it is necessary to accurately measure it in portfolio selection, underlying financial products pricing and hedging or risk management. Determining the implied volatility is a typical PDE inverse problem. It was first considered by Dupire in [4] where he obtained a formula of the local volatility with all possible strikes and maturities. However, the formula was ill-posed and could not be used in practice, it had to be modified. Some detailed treatments of problems in these areas can be found in [4-16]. In [5, 9, 15], Bouchouev and Isakov reduce the identification of volatility to an inverse problem with the final observation, and the local uniqueness and stability of volatility are proved under certain assumptions. Lu and $\mathrm{Yi} \mathrm{in} \mathrm{[12]} \mathrm{obtain} \mathrm{a} \mathrm{Fredholm} \mathrm{integral} \mathrm{equation} \mathrm{from} \mathrm{the} \mathrm{Dupire} \mathrm{equation.} \mathrm{In} \mathrm{[16],}$ Mishura consider the diffusion approximation for the recurrent schemes of financial markets and generalize a classical scheme of weak convergence for discrete-time markets to the Black-Scholes model. Based on the optimal control framework, in [10, 11], Jiang and Tao consider the inverse problem of determining the implied volatility $\sigma=\sigma(S)$ using the Tikhonov regularization strategy and analyze the existence and uniqueness of $\sigma=\sigma(S)$, and a new well-posed algorithm is presented. Similar results are obtained in [7], where a new extra condition (i.e., the average option premium) is assumed to be known. In [17], the authors also consider an optimal control problem to a mathematical model of drug treatment, a cost functional of the system is minimized and the total amount of drugs is given.

As we all know, Tikhonov regularization strategy is a widely applied method for solving the inverse problem (see $[13,18])$. A lot of research work has been carried out in recent years; see, for example, $[3,6,7,10,11,14,19-22]$ and the references therein. However, this method may over-smooth the exact solution, it cannot effectively approximate the original solution when the exact solution has some singularities. In image processing, this shortcoming will blur the edge. To overcome this weakness, in [23] Rudin proposed the 
total variation regularization strategy:

$$
\min _{\mu \in \Omega} \frac{\lambda}{2}\|\mu-f\|_{L^{2}(\Omega)}^{2}+|\nabla \mu|_{L^{1}(\Omega)}
$$

Based on the work of Rudin in [23], in recent years, the total variation regularization strategy has been applied to the problem of determining the volatility (see [13, 18, 24]). In $[13,24]$, Wang and Yang consider the inverse problem of identifying the implied volatility $\sigma=\sigma(S)$ using the total variation regularization strategy and analyze the existence and uniqueness of $\sigma=\sigma(S)$, the necessary condition which must be satisfied by the related minimum is also discussed, and the optimization algorithm is found using the limited memory quasi-Newton algorithm. The authors in [18] propose a bivariate total variation regularization model to calibrate the volatility $\sigma=\sigma(S, t)$, the existence and convergence for the regularized approach are discussed and some numerical experiments are presented.

Considering the advantages and great success of the total variation regularization in image processing and the work of $[13,18,23,24]$, in this paper, we would like to reconstruct the unknown volatility function $\sigma=\sigma(S)$ in (1.1) by using the total variation regularization strategy, based on the optimal control method (see $[6,7,13,17,22])$. Being different from the common inverse volatility problems arising in the area of option pricing (see $[6$, $7,11,13,16,18,20,22]$ ), our inverse problem has the following unusual features. First, in $[6,7,11,13,18,20,22]$ the underlying mathematical model is a semi-infinite initial value problem, whereas our model is an initial-boundary value parabolic equation defined on a bounded domain. Secondly, to reduce the inverse option pricing problem to an inverse parabolic problem with terminal observations, the conjugate theory (see [4]) should be adopted. For our problem, since the underlying asset has the property of double barrier option and the measurement data only depend on the price of gold, it is not necessary to use the Green function theory. Finally, the authors in $[6,7,10,11,22]$ use the Tikhonov regularization to identify the implied volatility, while it may over-smooth the exact solution and may not preserve the singularities of the solution well when the exact solution has some singularities. However, the total variation regularization model might be able to characterize the properties of the volatility better. Therefore, using our model to identify the implied volatility is a question worth thinking about. The inverse problem considered in the paper and the obtained results can be regarded as the beneficial supplement of the inverse option pricing problem.

The paper is organized as follows. In Section 2, we transform the parabolic problem (1.3) into an optimal control problem. In Section 3, the existence of the minimum for the control functional is given. The necessary condition which must be satisfied by the minimum is deduced in Section 4. In Section 5, we investigate the uniqueness of the minimum under some assumptions. The stability and convergence for the regularized approach are also presented in the last section.

\section{Optimal control problem}

Consider the following optimal control problem $\mathbf{P}$ :

Find $\bar{a} \in \mathcal{A}$ such that

$$
T(\bar{a})=\min _{a \in \mathcal{A}} T(a)
$$


where

$$
\begin{aligned}
& T(a)=\frac{1}{2} \int_{0}^{l}\left|U\left(x, \tau^{*}\right)-U^{*}(x)\right|^{2} d x+\frac{N}{2} J(a)+\frac{\mu}{2} G(a), \\
& J(a)=\int_{0}^{l}|\nabla a| d x, \quad G(a)=\int_{0}^{l}|\nabla a|^{2} d x, \\
& \mathcal{A}=\left\{a(x) \mid 0<\alpha \leq a(x) \leq \beta, \nabla a \in L^{2}(0, l)\right\},
\end{aligned}
$$

$U(x, \tau)$ is the solution of (1.3) for a given coefficient $a \in \mathcal{A}, N$ and $\mu$ are the regularization parameters. Considering the 'smile' or 'skew' effect of the implied volatility, it is reasonable to assume that $0<\alpha \leq a(x) \leq \beta$, where $\alpha$ and $\beta$ are two given positive constants. The implied volatility $a(x)$ is the principle coefficient in a second order parabolic equation. In general, it is required $a(x) \geq \alpha>0$. Moreover, for convenience, in the rest of discussion, it is also assumed to be bounded, i.e., $a(x) \leq \beta$. In practice, the parameter $\beta$ can be taken relatively larger. In fact, volatility is observed in many cases to be represented by two typical curves: smile and skew [4]. Many attempts have been made to remove this constant volatility assumption from the Black-Scholes model.

To avoid the case $|\nabla a|=0$, we replace $|\nabla a|$ with $|\nabla a|_{\epsilon}=\sqrt{|\nabla a|^{2}+\epsilon^{2}}$, where $\epsilon$ is a constant and $\epsilon \ll 1$. Then problem (2.1) is approximated by

$$
T(a)=\frac{1}{2} \int_{0}^{l}\left|U\left(x, \tau^{*}\right)-U^{*}(x)\right|^{2} d x+\frac{N}{2} \int_{0}^{l} \sqrt{|\nabla a|^{2}+\epsilon^{2}} d x+\frac{\mu}{2} \int_{0}^{l}|\nabla a|^{2} d x .
$$

This problem is different from the traditional Tikhonov regularization method because the regular item $\int_{0}^{l} \sqrt{|\nabla a|^{2}+\epsilon^{2}} d x$ is related to the total variation regularization.

\section{Existence}

Theorem 3.1 There exists a minimizer $\bar{a} \in \mathcal{A}$ of $T(a)$, i.e.,

$$
T(\bar{a})=\min _{a \in \mathcal{A}} T(a)
$$

Proof It is obvious that $T(a)$ is nonnegative and thus $T(a)$ has the greatest lower bound $\inf _{a \in \mathcal{A}} T(a)$. Let $\left(U_{n}, a_{n}\right)$ be a minimizing sequence, i.e.,

$$
\inf _{a \in \mathcal{A}} T(a) \leq T\left(a_{n}\right) \leq \inf _{a \in \mathcal{A}} T(a)+\frac{1}{n}, \quad n=1,2, \ldots
$$

By noticing that $T\left(a_{n}\right) \leq C$, and thanks to the particular structure of $T$, we can deduce that

$$
\left\|\nabla a_{n}\right\|_{L^{2}(0, l)} \leq C
$$

where $C$ is independent of $n$.

By the Sobolev imbedding theorem (see [25]), we can obtain

$$
\left\|a_{n}\right\|_{C^{\frac{1}{2}(0, l)}} \leq C \quad(C \text { is independent of } n) .
$$


Thus

$$
\begin{aligned}
& \left\|U_{n}(x, \tau)\right\|_{C^{\frac{1}{2}, \frac{1}{4}(\bar{Q})}} \leq C \quad(C \text { is independent of } n) \\
& \left\|U_{n}(x, \tau)\right\|_{C^{2+\frac{1}{2}, 1+\frac{1}{4}(\omega)}} \leq C, \quad \forall \omega \subset \subset Q
\end{aligned}
$$

where $Q=(0, l) \times\left[0, \tau^{*}\right]$.

Therefore we can select subsequences of $a_{n}$ and $U_{n}$, again denoted by $\left\{a_{n}\right\}$ and $\left\{U_{n}\right\}$, such that

$$
\begin{aligned}
& a_{n}(x) \rightarrow \bar{a}(x) \in C^{\frac{1}{2}}(0, l), \quad \text { uniformly in } C^{\alpha}(0, l)\left(0 \leq \alpha<\frac{1}{2}\right), \\
& U_{n}(x, \tau) \rightarrow \bar{U}(x, \tau), \quad \text { uniformly in } C^{\alpha, \frac{\alpha}{2}}(\bar{Q}) \cap C_{\mathrm{loc}}^{2+\alpha, 1+\frac{\alpha}{2}}(Q) .
\end{aligned}
$$

One can easily check that $(\bar{a}(x), \bar{U}(x, \tau))$ satisfies (1.3). By the Lebesgue control convergence theorem and the weak semi-continuity of the $L^{2}$-norm, we obtain

$$
T(\bar{a}) \leq \lim _{n \rightarrow \infty} \inf T\left(a_{n}\right)=\min _{a \in \mathcal{A}} J(a)
$$

Hence

$$
T(\bar{a})=\min _{a \in \mathcal{A}} T(a)
$$

This completes the proof of Theorem 3.1.

\section{Necessary condition}

Theorem 4.1 Let a be the solution of the optimal control problem (2.1). Then there exists a triple of functions $(U, \varphi, a)$ satisfying the following systems:

$$
\begin{aligned}
& \begin{cases}U_{\tau}-a(x)\left(U_{x x}-U_{x}\right)-r U_{x}+r U=f(x, \tau), & (x, \tau) \in(0, l) \times\left(0, \tau^{*}\right], \\
U(x, 0)=\phi(x), & x \in(0, l), \\
U(0, \tau)=U(l, \tau)=0, & \tau \in\left[0, \tau^{*}\right],\end{cases} \\
& \begin{cases}-\varphi_{\tau}-(a \varphi)_{x x}-(a \varphi)_{x}+r \varphi_{x}+r \varphi=0, & (x, \tau) \in(0, l) \times\left(0, \tau^{*}\right], \\
\varphi\left(x, \tau^{*}\right)=U\left(x, \tau^{*}\right)-U^{*}(x), & x \in(0,1), \\
\varphi(0, \tau)=\varphi(l, \tau)=0, & \tau \in\left[0, \tau^{*}\right],\end{cases}
\end{aligned}
$$

and

$$
\begin{aligned}
\int_{0}^{\tau^{*}} & \int_{0}^{l} \varphi(h-a)\left(U_{x x}-U_{x}\right) d x d \tau \\
+ & \frac{N}{2} \int_{0}^{l} \frac{\nabla a}{\sqrt{|\nabla a|^{2}+\epsilon^{2}}} \cdot \nabla(h-a) d x+\mu \int_{0}^{l} \nabla a \cdot \nabla(h-a) d x \geq 0
\end{aligned}
$$

for any $h \in \mathcal{A}$.

Proof For any $h \in \mathcal{A}, \delta \in[0,1]$, let

$$
a_{\delta}=(1-\delta) a+\delta h \in \mathcal{A} \text {. }
$$


Then we have

$$
\begin{aligned}
T_{\delta} & \equiv T\left(a_{\delta}\right) \\
& =\frac{1}{2} \int_{0}^{l}\left|U\left(x, \tau^{*} ; a_{\delta}\right)-U^{*}(x)\right|^{2} d x+\frac{N}{2} \int_{0}^{l} \sqrt{\left|\nabla a_{\delta}\right|^{2}+\epsilon^{2}} d x+\frac{\mu}{2} \int_{0}^{l}\left|\nabla a_{\delta}\right|^{2} d x .
\end{aligned}
$$

Let $U_{\delta}$ be the solution of (1.3) with given $a=a_{\delta}$. Since $a$ is an optimal solution, and thanks to the particular structure of $J$, we can deduce that the above function takes the minimum at $\delta=0$, so

$$
\begin{aligned}
\left.\frac{d T\left(a_{\delta}\right)}{d \delta}\right|_{\delta=0}= & \left.\int_{0}^{l}\left[U\left(x, \tau^{*} ; a\right)-U^{*}(x)\right] \frac{\partial U_{\delta}}{\partial \delta}\right|_{\delta=0} d x \\
& +\frac{N}{2} \int_{0}^{l} \frac{\nabla a}{\sqrt{|\nabla a|^{2}+\epsilon^{2}}} \cdot \nabla(h-a) d x+\mu \int_{0}^{l} \nabla a \cdot \nabla(h-a) d x \\
\geq & 0 .
\end{aligned}
$$

Let $U_{\delta}^{\prime} \equiv \frac{\partial U_{\delta}}{\partial \delta}$, direct calculations lead to the following equation:

$$
\begin{aligned}
& \frac{\partial}{\partial \tau}\left(U_{\delta}^{\prime}\right)=a_{\delta}\left(\frac{\partial^{2} U_{\delta}^{\prime}}{\partial x^{2}}-\frac{\partial U_{\delta}^{\prime}}{\partial x}\right)+r \frac{\partial U_{\delta}^{\prime}}{\partial x}-r U_{\delta}^{\prime}+(h-a)\left(\frac{\partial^{2} U_{\delta}}{\partial x^{2}}-\frac{\partial U_{\delta}}{\partial x}\right), \\
& \left.U_{\delta}^{\prime}\right|_{\tau=0}=0
\end{aligned}
$$

Let $\xi=\left.U_{\delta}^{\prime}\right|_{\delta=0}$, then $\xi$ satisfies

$$
\begin{aligned}
& \xi_{\tau}=a(x)\left(\xi_{x x}-\xi_{x}\right)+r \xi_{x}-r \xi+(h-a)\left(U_{x x}-U_{x}\right), \\
& \left.\xi\right|_{\tau=0}=0 .
\end{aligned}
$$

From (4.5) we have

$$
\begin{aligned}
& \int_{0}^{l}\left[U\left(x, \tau^{*} ; a\right)-U^{*}(x)\right] \xi\left(x, \tau^{*}\right) d x \\
& \quad+\frac{N}{2} \int_{0}^{l} \frac{\nabla a}{\sqrt{|\nabla a|^{2}+\epsilon^{2}}} \cdot \nabla(h-a) d x+\mu \int_{0}^{l} \nabla a \cdot \nabla(h-a) d x \geq 0 .
\end{aligned}
$$

Let

$$
\mathcal{L} \xi=\xi_{\tau}-a(x)\left(\xi_{x x}-\xi_{x}\right)-r \xi_{x}+r \xi
$$

and suppose $\varphi$ is the solution of the following problem:

$$
\left\{\begin{array}{l}
\mathcal{L}^{*} \varphi=-\varphi_{\tau}-(a \varphi)_{x x}-(a \varphi)_{x}+r \varphi_{x}+r \varphi=0 \\
\left.\varphi\right|_{\tau=\tau^{*}}=U\left(x, \tau^{*}\right)-U^{*}(x) \\
\varphi(0, \tau)=\varphi(l, \tau)=0
\end{array}\right.
$$

where $\mathcal{L}^{*}$ is the adjoint operator of the operator $\mathcal{L}$. 
From the Green formula, we have

$$
\begin{aligned}
\int_{0}^{\tau^{*}} & \int_{0}^{l}\left(\varphi \mathcal{L} \xi-\xi \mathcal{L}^{*} \varphi\right) d x d \tau \\
\quad= & \int_{0}^{\tau^{*}} \int_{0}^{l}\left[(\varphi \xi)_{\tau}-\left(a(x) \varphi \xi_{y}\right)_{y}+\left(\xi(a(x) \varphi)_{y}\right)_{y}+(\xi a(x) \varphi)_{y}-r(\xi \varphi)_{y}\right] d x d \tau \\
= & \left.\int_{0}^{l} \varphi \xi\right|_{(x, 0)} ^{\left(x, \tau^{*}\right)} d x=\int_{0}^{l}\left[U\left(x, \tau^{*} ; a\right)-U^{*}(x)\right] \xi\left(x, \tau^{*}\right) d x
\end{aligned}
$$

From (4.7) and (4.11), we can obtain

$$
\int_{0}^{l}\left[U\left(x, \tau^{*}\right)-U^{*}(x)\right] \xi\left(x, \tau^{*}\right) d x=\int_{0}^{\tau^{*}} \int_{0}^{l} \varphi(h-a)\left(U_{x x}-U_{x}\right) d x d \tau .
$$

Combining (4.8) and (4.12), one can easily obtain that

$$
\begin{aligned}
\int_{0}^{\tau^{*}} & \int_{0}^{l} \varphi(h-a)\left(U_{x x}-U_{x}\right) d x d \tau \\
& +\frac{N}{2} \int_{0}^{l} \frac{\nabla a}{\sqrt{|\nabla a|^{2}+\epsilon^{2}}} \cdot \nabla(h-a) d x+\mu \int_{0}^{l} \nabla a \cdot \nabla(h-a) d x \geq 0 .
\end{aligned}
$$

The proof of Theorem 4.1 is thus completed.

\section{Uniqueness}

It is well known that the optimal control problem is nonconvex. In general, one may not expect a unique solution. However, the local uniqueness of the minimizer for the control functional can be obtained if $\tau^{*} \ll 1$.

Suppose that $U_{1}^{*}(x)$ and $U_{2}^{*}(x)$ are two given functions which satisfy condition 4.1. Let $a_{1}(x)$ and $a_{2}(x)$ be two minimizers of the control problem (2.1) corresponding to $U_{i}^{*}(x)$ $(i=1,2)$, respectively, and $\left\{U_{i}, \varphi_{i}\right\}(i=1,2)$ be the solutions of system $(4.1) /(4.2)$ in which $\bar{a}=a_{i}(i=1,2)$, respectively.

Set

$$
U_{1}-U_{2}=u, \quad \varphi_{1}-\varphi_{2}=\Phi, \quad a_{1}-a_{2}=A
$$

Then $U$ and $\Phi$ satisfy the following equations:

$$
\begin{aligned}
& \left\{\begin{array}{l}
u_{\tau}-a_{1}(x)\left(u_{x x}-u_{x}\right)-r u_{x}+r u=A\left(U_{2 x x}-U_{2 x}\right), \\
u(x, 0)=0 \\
u(0, \tau)=u(l, \tau)=0 .
\end{array}\right. \\
& \left\{\begin{array}{l}
\frac{1}{a_{2}} \Phi_{\tau}+\Phi_{x x}+\Phi_{x}-r\left(\frac{\Phi}{a_{2}}\right)_{x}-\frac{r}{a_{2}} \Phi \\
\quad=\left(\frac{1}{a_{2}}-\frac{1}{a_{1}}\right)\left(a_{1} \varphi_{1}\right)_{\tau}-r\left[\left(\frac{1}{a_{2}}-\frac{1}{a_{1}}\right)\left(a_{1} \varphi_{1}\right)\right]_{x}-r\left(\frac{1}{a_{1}}-\frac{1}{a_{2}}\right)\left(a_{1} \varphi_{1}\right), \\
\Phi(x, 0)=0 \\
\Phi(0, \tau)=\Phi(l, \tau)=0 \\
\left.\Phi\right|_{\tau=\tau^{*}}=a_{1} U\left(x, \tau^{*}\right)+A\left(U_{2}\left(x, \tau^{*}\right)-U^{*}(x)\right) .
\end{array}\right.
\end{aligned}
$$


Lemma 5.1 For problem (4.2), we have the following estimate:

$$
\begin{aligned}
& \max _{\tau \in\left[0, \tau^{*}\right]} \int_{0}^{l} \varphi^{2} d x+\int_{0}^{\tau^{*}} \int_{0}^{l}\left|\varphi_{x}\right|^{2} d x d \tau \leq C \int_{0}^{l}\left|U\left(x, \tau^{*}\right)-U^{*}(x)\right|^{2} d x, \\
& \max _{\tau \in\left[0, \tau^{*}\right]} \int_{0}^{l}\left|\varphi_{x}\right|^{2} d x+\int_{0}^{\tau^{*}} \int_{0}^{l}\left|\varphi_{x x}\right|^{2} \leq C\left\|U\left(x, \tau^{*}\right)-U^{*}(x)\right\|_{H^{1}(0, l)}^{2}
\end{aligned}
$$

Lemma 5.2 For any bounded continuous function $g(x) \in C(0, l)$, we have

$$
\|g(x)\|_{\infty} \leq\left|g\left(x_{0}\right)\right|+\sqrt{l}\|\nabla g(x)\|_{L^{2}(0, l)},
$$

where $x_{0}$ is a fixed point in $(0, l)$.

Proof For $0<x<l$, we have

$$
\begin{aligned}
|g(x)| & \leq\left|g\left(x_{0}\right)\right|+\left|\int_{x_{0}}^{x} g^{\prime} d x\right| \\
& \leq\left|g\left(x_{0}\right)\right|+\left(\int_{0}^{l} 1 d x\right)^{\frac{1}{2}}\left(\int_{0}^{l}|\nabla g|^{2} d x\right)^{\frac{1}{2}} .
\end{aligned}
$$

This completes the proof of Lemma 5.2.

Lemma 5.3 For problem (5.1), we have the following estimate:

$$
\begin{aligned}
& \max _{\tau \in\left[0, \tau^{*}\right]} \int_{0}^{l} u^{2} d x \leq C(\max |A|)^{2} \int_{0}^{\tau^{*}} \int_{0}^{l}\left|U_{2 x x}-U_{2 x}\right|^{2} d x d \tau, \\
& \int_{0}^{\tau^{*}} \int_{0}^{l}\left|u_{x x}-u_{x}\right|^{2} d x d \tau \leq C(\max |A|)^{2} \int_{0}^{\tau^{*}} \int_{0}^{l}\left|U_{2 x x}-U_{2 x}\right|^{2} d x d \tau,
\end{aligned}
$$

where $C$ is a constant.

Proof From equation (5.1), we have, for $0<\tau \leq \tau^{*}$,

$$
\begin{aligned}
\int_{0}^{\tau} & \int_{0}^{l} a_{1}\left(u_{x x}-u_{x}\right)^{2} d x d \tau \\
= & \int_{0}^{\tau} \int_{0}^{l} u_{\tau}\left(u_{x x}-u_{x}\right) d x d \tau-\int_{0}^{\tau} \int_{0}^{l} A\left(U_{2 x x}-U_{2 x}\right)\left(u_{x x}-u_{x}\right) d x d \tau \\
& -r \int_{0}^{\tau} \int_{0}^{l} u_{x}\left(u_{x x}-u_{x}\right) d x d \tau+r \int_{0}^{\tau} \int_{0}^{l} u\left(u_{x x}-u_{x}\right) d x d \tau \\
= & \int_{0}^{\tau} \int_{0}^{l}\left(u_{\tau} u_{x}\right)_{x} d x d \tau-\int_{0}^{\tau} \int_{0}^{l} u_{\tau x} u_{x} d x d \tau \\
& -\int_{0}^{\tau} \int_{0}^{l} u_{\tau} u_{x} d x d \tau+r \int_{0}^{\tau} \int_{0}^{l} u_{x}^{2} d x d \tau \\
& +r \int_{0}^{\tau} \int_{0}^{l} u\left(u_{x x}-u_{x}\right) d x d \tau-\int_{0}^{\tau} \int_{0}^{l} A\left(U_{2 x x}-U_{2 x}\right)\left(u_{x x}-u_{x}\right) d x d \tau .
\end{aligned}
$$


By noticing the boundary conditions, we have

$$
\begin{aligned}
\int_{0}^{\tau} \int_{0}^{l} a_{1}\left(u_{x x}-u_{x}\right)^{2} d x d \tau \\
=-\int_{0}^{\tau} \int_{0}^{l} u_{x} u_{x \tau} d x d \tau-\int_{0}^{\tau} \int_{0}^{l} u_{\tau} u_{x} d x d \tau+r \int_{0}^{\tau} \int_{0}^{l} u_{x}^{2} d x d \tau \\
\quad+r \int_{0}^{\tau} \int_{0}^{l} u\left(u_{x x}-u_{x}\right) d x d \tau-\int_{0}^{\tau} \int_{0}^{l} A\left(U_{2 x x}-U_{2 x}\right)\left(u_{x x}-u_{x}\right) d x d \tau \\
=-\left.\frac{1}{2} \int_{0}^{l} u_{x}^{2}\right|_{(x, \tau)} d x-\int_{0}^{\tau} \int_{0}^{l} a_{1} u_{x}\left(u_{x x}-u_{x}\right) d x d \tau-\int_{0}^{\tau} \int_{0}^{l} A U_{x}\left(U_{2 x x}-U_{2 x}\right) d x d \tau \\
\quad-r \int_{0}^{\tau} \int_{0}^{l} u\left(u_{x x}-u_{x}\right) d x d \tau-\int_{0}^{\tau} \int_{0}^{l} A\left(U_{2 x x}-U_{2 x}\right)\left(u_{x x}-u_{x}\right) d x d \tau,
\end{aligned}
$$

where we have used the deformation of equation (5.1).

This yields

$$
\begin{aligned}
& \frac{1}{8} \int_{0}^{\tau} \int_{0}^{l} a_{1}\left(u_{x x}-u_{x}\right)^{2} d x d \tau+\left.\frac{1}{2} \int_{0}^{l} u_{x}^{2}\right|_{(x, \tau)} d x \\
& \leq 4 \max _{x \in(0, l)} a_{1}(x) \int_{0}^{\tau} \int_{0}^{l} u_{x}^{2} d x d \tau+C \int_{0}^{\tau} \int_{0}^{l} U^{2} d x d \tau \\
& \quad+C(\max |A|)^{2} \int_{0}^{\tau^{*}} \int_{0}^{l}\left|U_{2 x x}-U_{2 x}\right|^{2} d x d \tau
\end{aligned}
$$

By estimating (5.3) we have

$$
\begin{aligned}
& \frac{1}{8} \int_{0}^{\tau} \int_{0}^{l} a_{1}\left(u_{x x}-u_{x}\right)^{2} d x d \tau+\left.\frac{1}{2} \int_{0}^{l} u_{x}^{2}\right|_{(x, \tau)} d x \\
& \quad \leq 4 \max _{x \in(0, l)} a_{1}(x) \int_{0}^{\tau} \int_{0}^{l} u_{x}^{2} d x d \tau+C(\max |A|)^{2} \int_{0}^{\tau^{*}} \int_{0}^{l}\left|U_{2 x x}-U_{2 x}\right|^{2} d x d \tau .
\end{aligned}
$$

From Gronwall's inequality, we have

$$
\int_{0}^{\tau^{*}} \int_{0}^{l}\left(u_{x x}-u_{x}\right)^{2} d x d \tau+\int_{0}^{l} u_{x}^{2} d x \leq C(\max |A|)^{2} \int_{0}^{\tau^{*}} \int_{0}^{l}\left(U_{2 x x}-U_{2 x}\right)^{2} d x d \tau
$$

which implies the conclusion.

The proof of Lemma 5.3 is thus completed.

Lemma 5.4 For problem (5.2) we have the following estimate:

$$
\begin{aligned}
& \max _{\tau \in\left[0, \tau^{*}\right]} \int_{0}^{l} \Phi^{2} d x+\int_{0}^{\tau} \int_{0}^{l}\left|\Phi_{x}\right|^{2} d x d \tau \\
& \leq C(\max |A|)^{2} \int_{0}^{\tau^{*}} \int_{0}^{l}\left(\left|U_{2 x}\right|^{2}+\left|\varphi_{1 \tau}\right|^{2}\right) d x d \tau \\
& \quad+C(\max |A|)^{2} \int_{0}^{l}\left|U_{2}\left(x, \tau^{*}\right)-U^{*}(x)\right|^{2} d x
\end{aligned}
$$


Proof For problem (5.2), we have

$$
\begin{aligned}
\int_{\tau}^{\tau^{*}} & \int_{0}^{l}\left[\frac{1}{a_{2}} \Phi \Phi_{\tau}+\Phi \Phi_{x x}+\Phi \Phi_{x}-r \Phi\left(\frac{\Phi}{a_{2}}\right)_{x}-\frac{r}{a_{2}} \Phi^{2}\right] d x d \tau \\
= & \int_{\tau}^{\tau^{*}} \int_{0}^{l} \Phi\left[\left(\frac{1}{a_{2}}-\frac{1}{a_{1}}\right)\left(a_{1} \varphi_{1}\right)_{\tau}\right. \\
& \left.-r\left[\left(\frac{1}{a_{2}}-\frac{1}{a_{1}}\right)\left(a_{1} \varphi_{1}\right)\right]_{x}-r\left(\frac{1}{a_{1}}-\frac{1}{a_{2}}\right)\left(a_{1} \varphi_{1}\right)\right] d x d \tau
\end{aligned}
$$

This yields

$$
\begin{aligned}
\frac{1}{2 a_{1}} & \left.\int_{0}^{l} \Phi^{2}\right|_{(x, \tau)} d x+\int_{\tau}^{\tau^{*}} \int_{0}^{l}\left|\Phi_{x}\right|^{2} d x d \tau+\int_{\tau}^{\tau^{*}} \int_{0}^{l} \frac{r}{a_{2}} \Phi^{2} d x d \tau \\
= & \int_{0}^{l} \frac{1}{2 a_{2}}\left|a_{1} U\left(x, \tau^{*}\right)+A\left(U_{2}\left(x, \tau^{*}\right)-U^{*}(x)\right)\right|^{2} d x \\
& -\int_{\tau}^{\tau^{*}} \int_{0}^{l} \frac{A}{a_{2}} \Phi \varphi_{1 \tau} d x d \tau-\int_{\tau}^{\tau^{*}} \int_{0}^{l}\left(r \varphi_{1 x}+r \varphi_{1}\right) d x d \tau \\
\leq & C \int_{0}^{l}\left|U\left(x, \tau^{*}\right)\right|^{2}+C(\max |A|)^{2} \int_{0}^{l}\left|U_{2}\left(x, \tau^{*}\right)-U^{*}(x)\right|^{2} d x \\
& +\int_{\tau}^{\tau^{*}} \int_{0}^{l} \frac{1}{2 a_{2}} \Phi^{2} d x d \tau+C(\max |A|)^{2} \int_{\tau}^{\tau^{*}} \int_{0}^{l}\left|\varphi_{1 \tau}\right|^{2} d x d \tau \\
\leq & \int_{\tau}^{\tau^{*}} \int_{0}^{l} \frac{1}{2 a_{2}} \Phi^{2} d x d \tau+C(\max |A|)^{2} \int_{0}^{l}\left|U_{2}\left(x, \tau^{*}\right)-U^{*}(x)\right|^{2} d x \\
& +C(\max |A|)^{2} \int_{\tau}^{\tau^{*}} \int_{0}^{l}\left(\left|U_{2 x x}-U_{2 x}\right|^{2}+\left|\varphi_{1 \tau}\right|^{2}\right) d x d \tau .
\end{aligned}
$$

From (5.8) and Gronwall's inequality, we have

$$
\begin{aligned}
& \int_{0}^{l} \Phi^{2} d x+\int_{0}^{\tau^{*}} \int_{0}^{l} \Phi_{x}^{2} d x d \tau \\
& \leq C(\max |A|)^{2} \int_{0}^{l}\left|U_{2}\left(x, \tau^{*}\right)-U^{*}(x)\right|^{2} d x \\
& \quad+C(\max |A|)^{2} \int_{\tau}^{\tau^{*}} \int_{0}^{l}\left(\left|U_{2 x}\right|^{2}+\left|\varphi_{1 \tau}\right|^{2}\right) d x d \tau .
\end{aligned}
$$

This completes the proof of Lemma 5.4.

Theorem 5.1 Suppose that $a_{1}(x), a_{2}(x)$ are two minimizers of the optimal control problem (2.1) corresponding to $U_{1}^{*}(x)$ and $U_{2}^{*}(x)$, respectively. If there exists a point $x_{0} \in(0, l)$ such that $a_{1}\left(x_{0}\right)=a_{2}\left(x_{0}\right)$, then for $\tau^{*} \ll 1$, we have

$$
a_{1}(x) \equiv a_{2}(x), \quad \text { for any } x \in(0, l)
$$


Proof By taking $h=a_{2}$ when $\bar{a}=a_{1}$ and taking $h=a_{1}$ when $\bar{a}=a_{2}$ in (4.3), we have

$$
\begin{aligned}
& \int_{0}^{\tau^{*}} \int_{0}^{l} \varphi\left(a_{2}-a_{1}\right)\left(U_{1 x x}-U_{1 x}\right) d x d \tau \\
& \quad+\frac{N}{2} \int_{0}^{l} \frac{\nabla a_{1}}{\sqrt{\left|\nabla a_{1}\right|^{2}+\epsilon^{2}}} \cdot \nabla\left(a_{2}-a_{1}\right) d x+\mu \int_{0}^{l} \nabla a_{1} \cdot \nabla\left(a_{2}-a_{1}\right) d x \geq 0, \\
& \int_{0}^{\tau^{*}} \int_{0}^{l} \varphi\left(a_{1}-a_{2}\right)\left(U_{2 x x}-U_{2 x}\right) d x d \tau \\
& \quad+\frac{N}{2} \int_{0}^{l} \frac{\nabla a_{2}}{\sqrt{\left|\nabla a_{2}\right|^{2}+\epsilon^{2}}} \cdot \nabla\left(a_{1}-a_{2}\right) d x+\mu \int_{0}^{l} \nabla a_{2} \cdot \nabla\left(a_{1}-a_{2}\right) d x \geq 0,
\end{aligned}
$$

where $\left\{U_{i}, \varphi_{i}\right\}(i=1,2)$ are the solutions of system $(4.1) /(4.2)$ with $\bar{a}=a_{i}(i=1,2)$, respectively.

Combining equations (5.9) and (5.10), when $\epsilon \rightarrow 0$, we have

$$
\begin{aligned}
& \mu \int_{0}^{l}\left|\nabla\left(a_{1}-a_{2}\right)\right|^{2} d x \\
& \leq \int_{0}^{\tau^{*}} \int_{0}^{l} A\left(\varphi_{2}\left(U_{2 x x}-U_{2 x}\right)-\varphi_{1}\left(U_{1 x x}-U_{1 x}\right)\right) d x d \tau \\
& \leq \int_{0}^{\tau^{*}} \int_{0}^{l} \frac{1}{a_{2}} A\left(U_{2 x x}-U_{2 x}\right) a_{2} \varphi_{2} d x d \tau-\int_{0}^{\tau^{*}} \int_{0}^{l} \frac{1}{a_{1}} A\left(U_{1 x x}-U_{1 x}\right) a_{1} \varphi_{1} d x d \tau \\
& \leq \int_{0}^{\tau^{*}} \int_{0}^{l}\left(\frac{1}{a_{2}} A\left(U_{2 x x}-U_{2 x}\right) a_{2} \varphi_{2}-\frac{1}{a_{2}} A\left(U_{2 x x}-U_{2 x}\right) a_{1} \varphi_{1}\right) d x d \tau \\
& \quad+\int_{0}^{\tau^{*}} \int_{0}^{l}\left(\frac{1}{a_{2}} A\left(U_{2 x x}-U_{2 x}\right) a_{1} \varphi_{1}-\frac{1}{a_{2}} A\left(U_{1 x x}-U_{1 x}\right) a_{1} \varphi_{1}\right) d x d \tau \\
& \quad+\int_{0}^{\tau^{*}} \int_{0}^{l}\left(\frac{1}{a_{2}} A\left(U_{1 x x}-U_{1 x}\right) a_{1} \varphi_{1}-\frac{1}{a_{1}} A\left(U_{1 x x}-U_{1 x}\right) a_{1} \varphi_{1}\right) d x d \tau \\
& \leq \int_{0}^{\tau^{*}} \int_{0}^{l} \frac{1}{a_{2}} A^{2}\left(U_{1 x x}-U_{1 x}\right) \varphi_{1} d x d \tau \\
& \quad-\int_{0}^{\tau^{*}} \int_{0}^{l} \frac{1}{a_{2}} A \Phi\left(U_{2 x x}-U_{2 x}\right) d x d \tau \\
& \quad-\int_{0}^{\tau^{*}} \int_{0}^{l} \frac{1}{a_{2}} A\left(u_{x x}-u_{x}\right) a_{1} \varphi_{1} d x d \tau .
\end{aligned}
$$

From the assumption of Theorem 5.1, there exists a point $x_{0} \in(0, l)$ such that

$$
A\left(x_{0}\right)=a_{1}\left(x_{0}\right)-a_{2}\left(x_{0}\right)=0 .
$$

From Lemma 5.2 we have

$$
\max _{x \in[0, l]}|A| \leq C\left(\int_{0}^{l}|\nabla A|^{2} d x\right)^{1 / 2}
$$


From (5.3), (5.11) and Hölder's inequality, we have

$$
\begin{aligned}
& \mu \int_{0}^{l}|\nabla A|^{2} d x \\
& \leq C \max |A| \sqrt{\int_{0}^{\tau^{*}} \int_{0}^{l} \Phi^{2} d x d \tau} \cdot \sqrt{\int_{0}^{\tau^{*}} \int_{0}^{l}\left|U_{2 x x}-U_{2 x}\right|^{2} d x d \tau} \\
& +C \max |A|^{2} \sqrt{\int_{0}^{\tau^{*}} \int_{0}^{l}\left|\varphi_{1}\right|^{2} d x d \tau} \cdot \sqrt{\int_{0}^{\tau^{*}} \int_{0}^{l}\left|U_{1 x x}-U_{1 x}\right|^{2} d x d \tau} \\
& +C \max |A| \sqrt{\int_{0}^{\tau^{*}} \int_{0}^{l}\left|\varphi_{1}\right|^{2} d x d \tau} \cdot \sqrt{\int_{0}^{\tau^{*}} \int_{0}^{l}\left|u_{x x}-u_{x}\right|^{2} d x d \tau} \\
& \leq C \max |A|^{2} \sqrt{\tau^{*}\left(\int_{0}^{\tau^{*}} \int_{0}^{l}\left(\left|U_{2 x}\right|^{2}+\left|\varphi_{1 \tau}\right|^{2}\right) d x d \tau+\int_{0}^{l}\left|U_{2}\left(x, \tau^{*}\right)-U^{*}(x)\right|^{2} d x\right)} \\
& \sqrt{\int_{0}^{\tau^{*}} \int_{0}^{l}\left|U_{2 x x}-U_{2 x}\right|^{2} d x d \tau} \\
& +C \max |A|^{2} \sqrt{\int_{0}^{\tau^{*}} \int_{0}^{l}\left|\varphi_{1}\right|^{2} d x d \tau} \cdot \sqrt{\int_{0}^{\tau^{*}} \int_{0}^{l}\left|U_{1 x x}-U_{1 x}\right|^{2} d x d \tau} \\
& +C \max |A|^{2} \sqrt{\tau^{*} \int_{0}^{\tau^{*}} \int_{0}^{l}\left|\varphi_{1}\right|^{2} d x d \tau} \cdot \sqrt{\int_{0}^{\tau^{*}} \int_{0}^{l}\left|U_{2 x x}-U_{2 x}\right|^{2} d x d \tau}
\end{aligned}
$$

From Lemmas 5.1, 5.3 and 5.4, we have

$$
\begin{aligned}
& \int_{0}^{\tau^{*}} \int_{0}^{l}\left|U_{1 x x}-U_{1 x}\right|^{2} d x d \tau \leq C, \quad \int_{0}^{\tau^{*}} \int_{0}^{l}\left|U_{2 x x}-U_{2 x}\right|^{2} d x d \tau \leq C, \\
& \int_{0}^{\tau^{*}} \int_{0}^{l}\left|\varphi_{1}\right|^{2} d x d \tau \leq C \tau^{*}, \quad \int_{0}^{\tau^{*}} \int_{0}^{l} \varphi_{1 \tau}^{2} d x d \tau \leq C, \\
& \int_{0}^{l}\left|U_{2}\left(x, \tau^{*}\right)-U^{*}(x)\right|^{2} d x \leq C .
\end{aligned}
$$

Based on the above analysis, we have

$$
\mu \int_{0}^{l}|\nabla A|^{2} d x \leq C \max |A|^{2}\left(\sqrt{\tau^{*}}+\tau^{*}\right) \leq C\left(\sqrt{\tau^{*}}+\tau^{*}\right) \int_{0}^{l}|\nabla A|^{2} d x .
$$

Choose $\tau^{*} \leq 1$ such that

$$
\frac{C}{\mu}\left(\sqrt{\tau^{*}}+\tau^{*}\right)=\frac{1}{4}
$$

Combining (5.13) and (5.14), we can easily obtain

$$
\int_{0}^{l}|\nabla A|^{2} d x \leq 0
$$


This yields

$$
A(x)=a_{1}(x)-a_{2}(x)=a_{1}\left(x_{0}\right)-a_{2}\left(x_{0}\right) \equiv 0, \quad x \in(0, l) .
$$

This completes the proof of Theorem 5.1.

\section{Stability}

In the previous section, we have obtained the existence and uniqueness of the optimal solution. In this section, we will discuss the stability of the solution.

We suppose that the exact final observation $U^{*}(x)$ is attainable, i.e., there exists $a \in \mathcal{A}$ with $U(x, \tau ; a)=U^{*}(x)$, and that an upper bound $\sigma$ for the noise level

$$
\left\|U_{\sigma}^{*}(x)-U^{*}(x)\right\|_{L^{2}(0, l)} \leq \sigma
$$

of the observation is known a priori. Let $a_{\sigma} \in \mathcal{A}$ denote the solution to problem (2.1) with $U^{*}(x)$ replaced by $U_{\sigma}^{*}(x)$.

Theorem 6.1 The minimization of (2.1) is stable with respect to perturbations in the small data $\sigma$, i.e., if $U_{\sigma}^{*}(x) \rightarrow U^{*}(x)$, then we have the following estimate in $L^{2}(0, l)$ :

$$
a^{\sigma} \rightarrow a, \quad J_{\epsilon}\left(a_{\sigma}\right) \rightarrow J_{\epsilon}(a), \quad G\left(a_{\sigma}\right) \rightarrow G(a) .
$$

Proof $U_{\sigma}^{*}(x) \rightarrow U^{*}(x)$ in $L^{2}(\Omega)$ implies that $\left(a_{\sigma}, U_{\sigma}(x, \tau)\right)$ satisfies

$$
\begin{aligned}
& \frac{1}{2}\left\|U(x, \tau)-U_{\sigma}^{*}(x)\right\|_{L^{2}(0, l)}^{2}+\frac{N}{2} J_{\epsilon}\left(a_{\sigma}\right)+\frac{\mu}{2} G_{\epsilon}\left(a_{\sigma}\right) \\
& \quad \leq \frac{1}{2}\left\|U(x, \tau)-U_{\sigma}^{*}(x)\right\|_{L^{2}(0, l)}^{2}+\frac{N}{2} J_{\epsilon}(a)+\frac{\mu}{2} G_{\epsilon}(a) .
\end{aligned}
$$

Since $a_{\sigma}$ is bounded in $\mathcal{A}$, there exists a weakly convergent subsequence $\left\{a_{n}\right\}$ of $a_{\sigma}$ satisfying $\left\{a_{n}\right\} \rightarrow \widehat{a}$. Similarly, there exists a subsequence $\left\{U_{n}(x, \tau)\right\}$ corresponding to $\left\{a_{n}\right\}$ such that $\left\{U_{n}(x, \tau)\right\} \rightarrow \widehat{U}(x, \tau)$. By the weak semi-continuity of the $L^{2}$-norm, we have

$$
J_{\epsilon}(\widehat{a}) \leq \lim _{n \rightarrow \infty} \sup J_{\epsilon}\left(a_{n}\right)
$$

and

$$
\begin{aligned}
& \frac{1}{2}\left\|\widehat{U}(x, \tau)-U^{*}(x)\right\|_{L^{2}(0, l)}^{2}+\frac{\mu}{2}\|\nabla \widehat{a}\|_{L^{2}(0, l)} \\
& \quad \leq \limsup \left\{\frac{1}{2}\left\|U_{n}(x, \tau)-U_{n}^{*}(x)\right\|_{L^{2}(0, l)}^{2}\right\}+\frac{\mu}{2}\left\|\nabla a_{n}\right\|_{L^{2}(0, l)} .
\end{aligned}
$$

From (6.1), then for any $a \in L^{2}(0, l)$, we have

$$
\begin{aligned}
& \frac{1}{2}\left\|\widehat{U}(x, \tau)-U^{*}(x)\right\|_{L^{2}(0, l)}^{2}+\frac{N}{2} J_{\epsilon}(\widehat{a})+\frac{\mu}{2} G(\widehat{a}) \\
& \quad \leq \liminf \left\{\frac{1}{2}\left\|U_{n}(x, \tau)-U_{n}^{*}(x)\right\|_{L^{2}(0, l)}^{2}+\frac{N}{2} J_{\epsilon}\left(a_{n}\right)+\frac{\mu}{2} G\left(a_{n}\right)\right\}
\end{aligned}
$$




$$
\begin{aligned}
& \leq \lim \sup \left\{\frac{1}{2}\left\|U_{n}(x, \tau)-U_{n}^{*}(x)\right\|_{L^{2}(0, l)}^{2}+\frac{N}{2} J_{\epsilon}\left(a_{n}\right)+\frac{\mu}{2} G\left(a_{n}\right)\right\} \\
& \leq \lim _{n \rightarrow \infty}\left\{\frac{1}{2}\left\|U(x, \tau)-U_{n}^{*}(x)\right\|_{L^{2}(0, l)}^{2}+\frac{N}{2} J_{\epsilon}(a)+\frac{\mu}{2} G(a)\right\} \\
& =\frac{1}{2}\left\|U(x, \tau)-U^{*}(x)\right\|_{L^{2}(0, l)}^{2}+\frac{N}{2} J_{\epsilon}(a)+\frac{\mu}{2} G(a) .
\end{aligned}
$$

From this we can find that $\widehat{a}$ is a solution of the optimal control problem $\mathbf{P}$ and satisfies

$$
\begin{gathered}
\lim _{n \rightarrow \infty}\left\{\frac{1}{2}\left\|U_{n}(x, \tau)-U_{n}^{*}(x)\right\|_{L^{2}(0, l)}^{2}+\frac{N}{2} J_{\epsilon}\left(a_{n}\right)+\frac{\mu}{2} G\left(a_{n}\right)\right\} \\
=\frac{1}{2}\left\|\widehat{U}(x, \tau)-U^{*}(x)\right\|_{L^{2}(0, l)}^{2}+\frac{N}{2} J_{\epsilon}(\widehat{a})+\frac{\mu}{2} G(\widehat{a}) .
\end{gathered}
$$

If $\left\{a_{n}\right\}$ does not converge to $\widehat{a}$, then

$$
\begin{aligned}
& \frac{1}{2}\left\|\widehat{U}(x, \tau)-U^{*}(x)\right\|_{L^{2}(0, l)}^{2}+\frac{\mu}{2} G(\widehat{a}) \\
& \quad<\lim \sup \left\{\frac{1}{2}\left\|U_{n}(x, \tau)-U^{*}(x)\right\|_{L^{2}(0, l)}^{2}+\frac{N}{2} J_{\epsilon}\left(a_{n}\right)+\frac{\mu}{2} G\left(a_{n}\right)\right\}=: C,
\end{aligned}
$$

and there exists a subsequence $a_{m}$ of $a_{n}$ satisfying

$$
\begin{aligned}
& a_{m} \rightarrow \widehat{a}, \quad U_{m} \rightarrow \widehat{U}, \quad J_{\epsilon}(\widehat{a}) \leq \lim _{m \rightarrow \infty} J_{\epsilon}\left(a_{m}\right), \\
& \frac{1}{2}\left\|U_{m}(x, \tau)-U^{*}(x)\right\|_{L^{2}(0, l)}^{2}+\frac{\mu}{2} G\left(a_{m}\right) \rightarrow C .
\end{aligned}
$$

From (6.2) we have

$$
\frac{N}{2} \lim _{m \rightarrow \infty} J_{\epsilon}\left(a_{m}\right)=\frac{N}{2} J_{\epsilon}(\widehat{a})+\frac{1}{2}\left\|\widehat{U}(x, \tau)-U^{*}(x)\right\|_{L^{2}(0, l)}^{2}+\frac{\mu}{2} G(\widehat{a})-C<\frac{N}{2} J_{\epsilon}(\widehat{a})
$$

which is a contradiction to (6.3). This shows that

$$
a_{m} \rightarrow \widehat{a}, \quad \lim _{m \rightarrow \infty} J_{\epsilon}\left(a_{m}\right)=J_{\epsilon}(\widehat{a}), \quad G\left(a_{m}\right) \rightarrow G(\widehat{a})
$$

The proof of Theorem 6.1 is thus completed.

\section{Concluding remarks}

A lot of research works have been made to identify the implied volatility by regularization methods. In this paper, we propose the total variation regularization strategy for solving the implied volatility in the Black-Scholes model. Based on the optimal control framework, the inverse problem of determining the implied volatility in financial products linked with gold price is discussed, which is still an interesting issue in financial mathematics. Since the profit of the derivative product depends not only on some cumulative indexes of gold price but also on some trigger indexes, such kind of product is, in a sense, similar to the double barrier option. So, it is quite meaningful to reconstruct the volatility function by the information obtained from the financial market. 
The difficulty is due to the lack of conventional stability, nonlinearity and nonconvexity. Based on the optimal control framework, the inverse problem is reduced to an optimization problem, then we propose the total variation regularization for identifying the implied volatility, and the existence, uniqueness and stability of the minimizer are proved. The results obtained in the paper are interesting and useful and may be applied to a variety of derivatives pricing problems.

\begin{abstract}
Acknowledgements
We would like to thank the editor and anonymous referees for their valuable comments and helpful suggestions, which improved the earlier version of the manuscript. This work is supported by the National Natural Science Foundation of China (No. 11261029, 11461039).
\end{abstract}

\title{
Competing interests
}

None of the authors has any competing interests as regards the manuscript.

\section{Authors' contributions}

All authors read and approved the final version of the manuscript.

\section{Publisher's Note}

Springer Nature remains neutral with regard to jurisdictional claims in published maps and institutional affiliations.

Received: 27 June 2017 Accepted: 2 January 2018 Published online: 15 January 2018

\section{References}

1. Jiang, LS: Mathematical Modeling and Methods of Option Pricing. Higher Education Press, Beijing (2008)

2. Jiang, LS, Xu, CL, Ren, XM, Li, SH: Mathematical Models and Cases Analysis of Financial Derivatives Pricing. Higher Education Press, Beijing (2008)

3. Engl, HW, Hanke, M, Neubauer, A: Regularization of Inverse Problems. Kluwer Academic, Dordrecht (1996)

4. Dupire, B: Pricing with a smile. Risk 7(1), 18-20 (1994)

5. Bouchouev, I, Isakov, V: Uniqueness, stability and numerical methods for the inverse problem that arises in financial markets. Inverse Probl. 15, R95-R116 (1999)

6. Yang, L, Deng, ZC: An inverse radiative coefficient problem arising in a two-dimensional heat conduction equation with a homogeneous Dirichlet boundary condition in a circular section. J. Math. Anal. Appl. 435, 917-943 (2016)

7. Deng, ZC, Yu, JN, Yang, L: An inverse problem of determining the implied volatility in option pricing. J. Math. Anal. Appl. 340, 16-31 (2008)

8. Isakov, V: Inverse Problems for Partial Differential Equations. Springer, New York (1998)

9. Isakov, V: Recovery of time dependent volatility coefficient by linearization. Evol. Equ. Control Theory 3, 119-134 (2014)

10. Jiang, LS, Chen, QH, Wang, LJ, Zhang, JE: A new well-posed algorithm to recover implied local volatility. Quant. Finance 4, 451-457 (2003)

11. Jiang, LS, Tao, YS: Identifying the volatility of underlying assets from option prices. Inverse Probl. 17, 137-155 (2001)

12. Lu, L, Yi, L: Recover implied volatility of underlying asset from European option price. J. Inverse III-Posed Probl. 17 , 499-509 (2009)

13. Wang, SL, Yang, YF: The total variation model for determining the implied volatility in option pricing. J. Comput. Appl. Math. 17(1), 111-124 (2014)

14. Hoa, NV, Khanh, TQ: Two-parameter regularization method for an axisymmetric inverse heat problem. Bound. Value Probl. 2017, Article ID 25 (2017)

15. Bouchouev, I, Isakov, V: The inverse problem of option pricing. Inverse Probl. 13, 7-11 (1997)

16. Mishura, Y: Diffusion approximation of recurrent schemes for financial markets, with application to the Ornstein-Uhlenbeck process. Opusc. Math. 35(1), 99-116 (2015)

17. Leszczyński, M, Ratajczyk, E, Ledzewicz, U, Schättler, H: Sufficient conditions for optimality for a mathematical model of drug treatment with pharmacodynamics. Opusc. Math. 37(3), 403-419 (2017)

18. Zeng, YH, Wang, SL, Yang, YF: Calibration of the volatility in option pricing using the total variation regularization. J. Appl. Math. 2014, Article ID 510819 (2014)

19. Deng, ZC, Yang, L, Yu, JN, Luo, GW: Identifying the diffusion coefficient by optimization from the final observation. Appl. Math. Comput. 219, 4410-4422 (2013)

20. Chen, Q, Liu, JJ: Solving an inverse parabolic problem by optimization from final measurement data. J. Comput. Appl. Math. 193, 183-203 (2006)

21. Sakthivel, K, Gnanavel, S: Inverse problem for the reaction diffusion system by optimization method. Appl. Math. Model. 35, 571-579 (2011)

22. Yang, L, Yu, JN, Deng, ZC: An inverse problem of identifying the coefficient of parabolic equation. Appl. Math. Model. 32, 1984-1995 (2008)

23. Rudin, L, Osher, S, Fatemi, E: Nonlinear total variation based noise removal algorithms. Physica D 60, 259-268 (1992)

24. Zeng, YH, Wang, SL, Yang, YF: The adjoint method for the inverse problem of option pricing. Math. Probl. Eng. 2014, Article ID 314104 (2014)

25. Adams, RA: Sobolev Spaces. Academic Press, New York (1975) 